
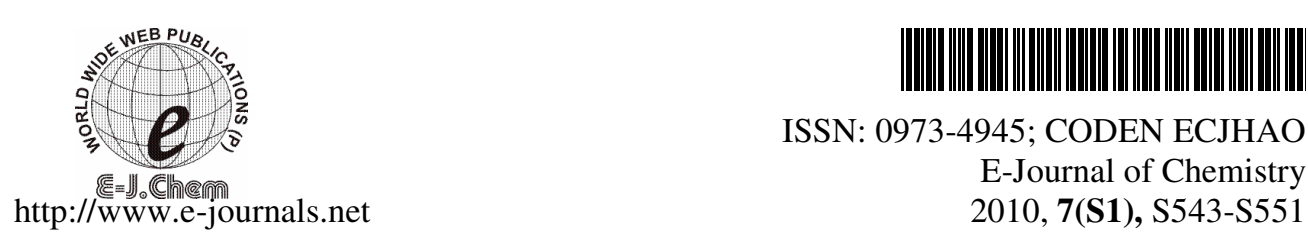

ISSN: 0973-4945; CODEN ECJHAO

E-Journal of Chemistry

2010, 7(S1), S543-S551

\title{
Microwave Assisted Synthesis of Indole Substituted Alkenes Using Knoevenagel Condensation Reaction and their Antibacterial Activity Study
}

\author{
SHUBHA JAIN* ${ }^{*}$ B. NAGI REDDY, K. SAMBASIVA RAO ${ }^{\#}$ and G. NEELIAH \\ Laboratory of Heterocycles, School of Studies in Chemistry \& Biochemistry \\ Vikram University, Ujjain, Madhya Pradesh-456010, India \\ ${ }^{\#}$ CavinKare Research Centre, Chennai, Tamilnadu- 600032, India \\ drshubhajain@yahoo.co.in
}

Received 25 February 2010; Accepted 1 May 2010

\begin{abstract}
The reaction rate of Knoevenagel condensation can be dramatically enhanced by irradiating the reaction mixture containing an aldehyde, active methylene compound, $L$-proline and chlorobenzene with a commercial microwave oven. Twenty Knoevenagel condensation products were synthesized within 5-17 min in good yields. The new compounds were also screened for their antibacterial activity.
\end{abstract}

Keywords: Active methylene compounds, Aldehydes, Substituted alkenes, Knoevenagel reaction.

\section{Introduction}

The importance of indoles is well recognized by synthetic as well as biological chemists. The most ubiquitous of the known bioactive alkaloids are based on the indole moiety. Medicinal chemists repeatedly turn to indole based compounds as a target pharmacophore for the development of therapeutic agents ${ }^{1}$. Nitrogen heterocycles are ubiquitous systems in nature and are consequently considered as privileged structures in drug discovery. Derivatisation of these heterocyclic pharmacophores represents a convenient approach to generate chemical diversity during lead identification and optimization ${ }^{2-4}$.

In the recent years the use of microwave irradiation in organic reactions is rapidly increasing, because of the short reaction time and the operational simplicity. It has been reported that a variety of reactions such as Diels-Alder ${ }^{5}$, ene $^{6}$, Claisen reactions ${ }^{7}$, Fischer cyclization $^{8}$, synthesis of heterocycles ${ }^{9}$, hydrolysis of esters ${ }^{10}$, phosphoric anhydride $^{11}$ and adenosine triphosphate ${ }^{12}$, hydrogenation ${ }^{13}$, deprotection of benzyl esters ${ }^{14}$, deacetylation of diacetates ${ }^{15}$, Graebe-Ullmann synthesis ${ }^{16}$ and oxazoline formation ${ }^{17}$, could be facilitated by microwave irradiation in a good energy transferring medium. 
We found that the reaction rate of the Knoevenagel condensation could be enhanced by the microwave irradiation. In the present study, four substituted indole aldehydes viz. 5-cyanoindole-3-carbaldehyde (1a), 5-bromoindole-3-carbaldehyde (1b), 1-methyl indole-3carbaldehyde (1c) and indole-3-carbaldehyde (1d) have been condensed with five active methylene compounds namely malononitrile (2a), ethyl cyanoacetate (2b), meldrum`s acid (2c), barbituric acid (2d) and dimidone (2e) using $L$-proline as base are mentioned in Table 1 . A large vial with a loose cap or an Erlenmeyer flask with a funnel as a loose top was used as the reaction vessel for the condensation. Mono chlorobenzene was used as an energy-transfer medium since its boiling point $\left(131-133{ }^{\circ} \mathrm{C}\right)$ is about $30{ }^{\circ} \mathrm{C}$ higher than water which is to be eliminated in the process. All the reactions were completed within 5-17 min. To the best of our knowledge, there are no reports for the synthesis of indole substituted alkenes by Knoevenagel condensation under microwave irradiation.

\section{Experimental}

IR spectra were recorded on a spectrum BX Series. ${ }^{1} \mathrm{H} \&{ }^{13} \mathrm{C}$ NMR spectra were recorded on $400 \mathrm{MHZ}$ spectrometer in DMSO- $\mathrm{d}_{6}$ using TMS as internal standard. Mass spectra were recorded on a DART mass spectrometer. M.ps. were taken on a Haake Bucher meting point apparatus and are uncorrected.

\section{General synthetic procedure}

To a solution of malononitrile $2 \mathbf{a}(2.2 \mathrm{mmol})$ in mono chlorobenzene and the catalyst $(0.2 \mathrm{mmol})$ was added indole aldehyde $1 \mathbf{a}(2 \mathrm{mmol})$ rapidly and all at once. The resulting reaction mixture was then irradiated in the microwave oven for $5 \mathrm{~min}$. The progress of the reaction was monitored by TLC. After complete conversion of the starting material as indicated by TLC, the reaction mixture was quenched with water and the solid produced was isolated by simple filtration and dried. The solid product 3a was identified by spectroscopic measurements and by comparison with an authentic samples and needed no further purification. Similarly, utilizing the aldehydes 1a-d and active methylene compounds 2a-e, compounds $\mathbf{3 b}$-t were synthesized and characterized (Table 1).

Table1. Synthesis of indole substituted alkenes from the Knoevenagel condensation of aldehydes and active methylene compounds

\begin{tabular}{|c|c|c|c|c|c|c|}
\hline R1 of 1 & $\mathrm{R} 2$ of 1 & 2 & Product & Reaction time, min & Yield, \% & M.P. ${ }^{0} \mathrm{C}$ \\
\hline $\mathrm{CN}$ & $\mathrm{H}$ & & $3 \mathbf{a}$ & 5 & 96 & 289-291 \\
\hline $\mathrm{CN}$ & $\mathrm{H}$ & & $3 \mathbf{b}$ & 8 & 82 & $276-278$ \\
\hline $\mathrm{CN}$ & $\mathrm{H}$ & & $3 c$ & 15 & 82 & 296-299 \\
\hline $\mathrm{CN}$ & $\mathrm{H}$ & & 3d & 16 & 76 & 263-264 \\
\hline
\end{tabular}




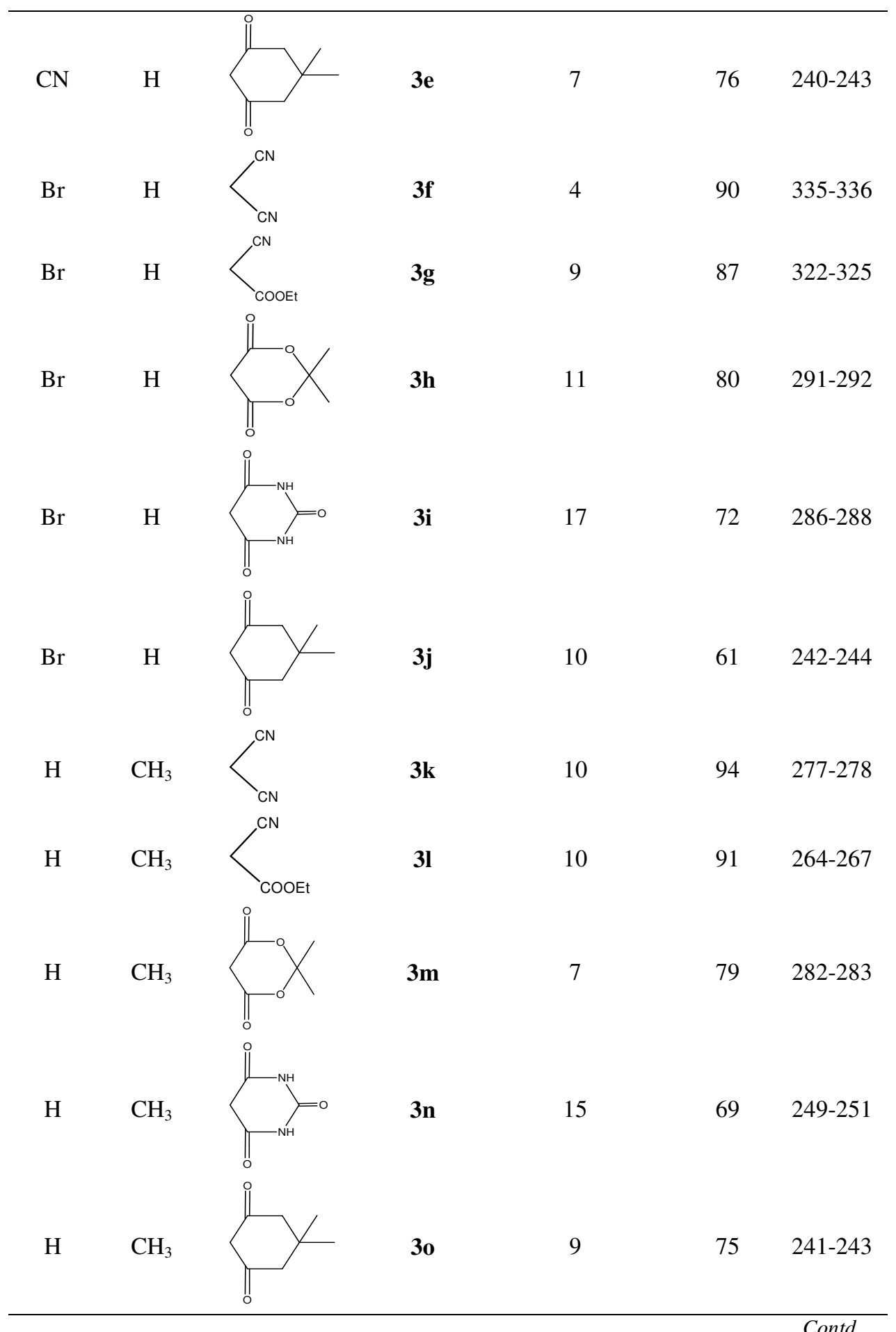

Contd... 


\begin{tabular}{|c|c|c|c|c|c|}
\hline $\mathrm{H}$ & $\mathrm{H}$ & $3 p$ & 5 & 89 & $\begin{array}{l}187- \\
189^{18}\end{array}$ \\
\hline $\mathrm{H}$ & $\mathrm{H}$ & $3 q$ & 5 & 82 & $\begin{array}{l}163- \\
166^{19} \\
(160- \\
162)\end{array}$ \\
\hline $\mathrm{H}$ & $\mathrm{H}$ & $3 \mathbf{r}$ & 15 & 74 & $175-176$ \\
\hline $\mathrm{H}$ & $\mathrm{H}$ & $3 s$ & 10 & 65 & $183-187$ \\
\hline $\mathrm{H}$ & $\mathrm{H}$ & $3 t$ & 8 & 71 & $162-163$ \\
\hline
\end{tabular}

\section{Results and Discussion}

In our continued interest in the development of a highly expedient methodology for the synthesis of fine chemicals and heterocyclic compounds of biological importance, we report here the first example of the synthesis of indole substituted alkenes from the Knoevenagel condensation of various indole substituted aldehydes (1) and active methylene compounds (2), for example, malononitrile, ethyl cyanoacetate, barbituric acid, meldrum`s acid and dimedone under microwave irradiation (Scheme 1). It was exciting to observe that, cyano group substituted active methylene compounds are more reactive than other active methylene compounds giving excellent yields of Knoevenagel products (3).

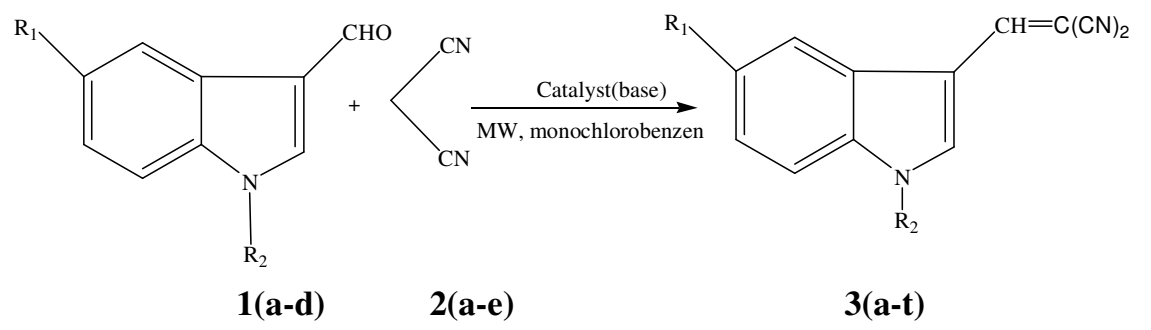

Scheme 1.

Spectral data of some of the representative compounds

2-((5-Cyano-3-indolyl)methylene)malononitrile (3a)

Yellow Solid: M.P. 289-291 ${ }^{0} \mathrm{C}$; (Found: C, 70.55; H, 2.56; N, 25.60. $\mathrm{C}_{13} \mathrm{H}_{6} \mathrm{~N}_{4}$ requires C 71.55; H, 2.77; N, 25.68; IR (KBr) 3270, 2915, 2843, 2215, 1566, 1495, 1336, 1225, 1139 , $825,789 \mathrm{~cm}^{-1} ;{ }^{1} \mathrm{H}$ NMR (400 MHz, DMSO-d 6 ) 7.66(d, 1H, $\left.12 \mathrm{~Hz}\right), 7.75(\mathrm{~d}, 1 \mathrm{H}, 12 \mathrm{~Hz})$, 
8.44(s,1H), 8.66(s, 1H, NH-CH=C),9.55(s, $\left.1 \mathrm{H},-\mathrm{CH}=\mathrm{C}(\mathrm{CN})_{2}, 11.20(\mathrm{~s}, 1 \mathrm{H}, \mathrm{NH}) ;\right) ;{ }^{13} \mathrm{C} \mathrm{NMR}$ (50 MHz): 94.02, 110.21,102.92, 114.26,114.72, 114.82(2-CN),122.42, 126.72, 128.25 , 135.22, 135.62, 146.32.;ES-MS $m / z$ 218( $\left.\mathrm{M}^{+}, 100 \%\right)$.

Ethyl(E)-2-cyano(5-cyano-3-indol)propenoate (3b)

Yellow Needles: M.P.276-278 ${ }^{0}$ C; (Found): C, 67.89; H, 4.16; N, 15.79; O, 12.06. $\mathrm{C}_{15} \mathrm{H}_{11} \mathrm{~N}_{3} \mathrm{O}_{2}$ requires C 67.92; $\mathrm{H}, 4.18 ; \mathrm{N}, 15.84 ; \mathrm{O}, 12.08$; $\mathrm{IR}(\mathrm{KBr}) 3400-3200,3100-2900$, 2210, 1670, 1600, 1590,1240, $1100 \mathrm{~cm}^{-1} ;{ }^{1} \mathrm{H}$ NMR (400 MHz, DMSO-d $\left.\mathrm{d}_{6}\right) 1.3(\mathrm{t}, 3 \mathrm{H}, 9.6 \mathrm{~Hz}$, $\left.\mathrm{CH}_{3}\right), 4.3\left(\mathrm{q}, 2 \mathrm{H}, 9.2 \mathrm{~Hz}, \mathrm{CH}_{2}\right), 7.65(\mathrm{~d}, 1 \mathrm{H}, 12 \mathrm{~Hz}), 7.74(\mathrm{~d}, 12 \mathrm{H}, 1 \mathrm{H}), 8.42(\mathrm{~s}, 1 \mathrm{H}), 8.65(\mathrm{~s}, 1 \mathrm{H}, \mathrm{NH}-$ $\mathrm{CH}=\mathrm{C}), 9.01\left(\mathrm{~s}, 1 \mathrm{H},-\mathrm{CH}=\mathrm{C}(\mathrm{CN})_{2}, 11.10(\mathrm{~s}, 1 \mathrm{H}, \mathrm{NH}) ;{ }^{13} \mathrm{C} \mathrm{NMR}(50 \mathrm{MHz}) 14.13\left(\mathrm{CH}_{3}\right), 61.46\right.$ $\left(\mathrm{OCH}_{2}\right), 93.42,102.2,110.22,113.83,114.81(\mathrm{CN}), 114.91,121.33,126.16,128.62,135.02$, 135.25, 146.24, 163.01(CO);ES-MS $m / z, 265\left(\mathrm{M}^{+}, 100 \%\right)$.

3-((2,2-Dimethyl-4,6-dioxo-1,3-dioxan-5-ylidene)methyl)indole-5-carbonitrile (3c)

Yellow solid: M.P.296-299 ${ }^{0} \mathrm{C}$; (Found): C, 64.83; H, 4.02; N, 9.41; O, 21.57. $\mathrm{C}_{16} \mathrm{H}_{12} \mathrm{~N}_{2} \mathrm{O}_{4}$ requires C 64.86; H, 4.08; N, 9.46; O, 21.60; IR (RBr) 3400-3200, 3100-2910, 2232,1700, 1670, 1600, 1594, 1240, $1108 \mathrm{~cm}^{-1}$; ${ }^{1} \mathrm{H}$ NMR (400 MHz, DMSO-d 6 ) 1.33(s, 6H), 7.67(d, 1H, $12 \mathrm{~Hz}), 7.73(\mathrm{~d}, 1 \mathrm{H}, 12 \mathrm{~Hz}), 7.98(\mathrm{~s}, 1 \mathrm{H}), 8.66(\mathrm{~s}, 1 \mathrm{H} \mathrm{NH}-\mathrm{CH}=\mathrm{C}), 8.7(\mathrm{~s}, 1 \mathrm{H}, \mathrm{CH}=\mathrm{C}), 11.22$ $(\mathrm{s}, 1 \mathrm{H}, \mathrm{NH}) ;{ }^{13} \mathrm{C}$ NMR $(50 \mathrm{MHz}): 15.2\left(2 \mathrm{CH}_{3}\right), 102.91,107.25,110.27,113.92,114.12$, $122.12,125.92,126.21,128.72,135.22,136.57,145.92,164.64 \quad(2 \mathrm{CO}) ;$ ES-MS $\mathrm{m} / \mathrm{z}$ 296( $\left.\mathrm{M}^{+}, 100 \%\right)$.

\section{3-((2,4,6-Trioxo-tetrahydropyrimidin-5 ylidene)methyl)indole-5-carboni trile (3d)}

Yellow solid: M.P. 263-264 ${ }^{0} \mathrm{C}$; (Found): C, 59.99; H, 2.83; N, 19.92; O, 17.11. $\mathrm{C}_{14} \mathrm{H}_{8} \mathrm{~N}_{4} \mathrm{O}_{3}$ requires $\mathrm{C} 60.00 ; \mathrm{H}, 2.88$; N, 19.99; O, 17.13; IR (RBr) 3400-3200, 31002900, 2210, 1670, 1600, 1590,1240, $1100 \mathrm{~cm}^{-1} ;{ }^{1} \mathrm{H}$ NMR (400 MHz, DMSO-d $)$ ) 7.52(d, $1 \mathrm{H}, 12 \mathrm{~Hz}), \quad 7.59(\mathrm{~d}, 1 \mathrm{H}, 12 \mathrm{~Hz}), 7.92(\mathrm{~s}, 1 \mathrm{H}), 8.65(\mathrm{~s}, 1 \mathrm{H}, \mathrm{NH}-\mathrm{CH}=\mathrm{C}), 8.72(\mathrm{~s}, 1 \mathrm{H}$, $\mathrm{CH}=\mathrm{C}), 11.22(\mathrm{~s}, 1 \mathrm{H}, \mathrm{NH}), 11.31(\mathrm{~s}, 2 \mathrm{H}, \mathrm{NH}-\mathrm{C}=\mathrm{O}) ;{ }^{13} \mathrm{C}$ NMR $(50 \mathrm{MHz}): 102.32,110.54$, 113.12 , 113.93, 123.02, 124.06, 125.06, 126.24, 135.21, 136.36, 147.22, 152.42,160.02 (2CO); ES-MS $m / z$ 280( $\left.\mathrm{M}^{+}, 100 \%\right)$.

\section{3-((4,4-Dimethyl-2,6-dioxocyclohexylidene)methyl)indole-5-carbonitrile(3e)}

Yellow solid: M.P. $240-243{ }^{\circ} \mathrm{C}$; (Found): C, 73.92; H, 5.50; N, 9.57; O, 10.91. $\mathrm{C}_{18} \mathrm{H}_{16} \mathrm{~N}_{2} \mathrm{O}_{2}$ requires C 73.95; H, 5.52; N, 9.58; O, 10.95; IR (KBr) 3400-3200, 3070, 3040,2964, 2935, 2870, 2232,1710, 1673, 1600, 1592,1423, 1410, $1100 \mathrm{~cm}^{-1 ; 1} \mathrm{H}$ NMR (400 MHz, DMSO$\left.\mathrm{d}_{6}\right), 1.31(\mathrm{~s}, 6 \mathrm{H}), \quad 2.24(\mathrm{~s}, 4 \mathrm{H}), 7.65(\mathrm{~d}, 1 \mathrm{H}, 12 \mathrm{~Hz}), 7.72(\mathrm{~d}, 1 \mathrm{H}, 12 \mathrm{~Hz}), 7.96(\mathrm{~s}, 1 \mathrm{H}), 8.6(\mathrm{~s}, 1 \mathrm{H}$, $\mathrm{CH}=\mathrm{C}), 8.64(\mathrm{~s}, 1 \mathrm{H}, \mathrm{NHCH}=\mathrm{C}), 11.20(\mathrm{~s}, 1 \mathrm{H}, \mathrm{NH}) ;{ }^{13} \mathrm{C} \mathrm{NMR}(50 \mathrm{MHz}): 14.72\left(2 \mathrm{CH}_{3}\right), 33.43$, 50.28, 103.01,110.52,112.96,114.02,122.91,126.24, 128.16, 128.40, 133.65, 135.01, 146.52, 163.02 (2CO); ES-MS m/z 292(M+100\%).

\section{2-((5-Bromo-3 indolyl)methylene)malononitrile (3f)}

Yellow solid:M.P. 335-336 ${ }^{0} \mathrm{C}$; (Found): C, 52.95; H, 2.21; Br, 29.35, N, 15.41 . $\mathrm{C}_{12} \mathrm{H}_{6} \mathrm{BrN}_{3}$ requires C 52.97; H, 2.22; Br, 29.37; N, 15.44; IR (KBr) 3270, 2915, 2843, 2215, 1566, 1495, 1336, 1225, 1139, 825, 789 $\mathrm{cm}^{-1} ;{ }^{1} \mathrm{H}$ NMR (400 MHz, DMSO-d $\left.\mathrm{d}_{6}\right)$ 7.42(d, 1H, $8.4 \mathrm{~Hz}), 7.54(\mathrm{~d}, 1 \mathrm{H}, 8.4 \mathrm{~Hz}), 7.98(\mathrm{~s}, 1 \mathrm{H}), 8.58(\mathrm{~s}, 1 \mathrm{H}, \mathrm{N}-\mathrm{CH}=\mathrm{C}), 9.50(\mathrm{~s}$, $\left.1 \mathrm{H}, \mathrm{CH}=\mathrm{C}(\mathrm{CN})_{2}\right), 11.2(\mathrm{~s}, 1 \mathrm{H}, \mathrm{NH}) ;{ }^{13} \mathrm{C} \mathrm{NMR}(50 \mathrm{MHz}): 94.22,109.55,115.26$, 115.62(2-CN), 118.22, 121.42, 127.20, 128.72, 133.35, 134.98, 147.22.; ES-MS m/z $270\left(\mathrm{M}^{+}, 100 \%\right)$. 


\section{Ethyl 3-(5-Bromo-3-indolyl) 2 cyanoacrylate (3g)}

Yellow Needles: M.P. $322-325{ }^{\circ}$ C; (Found): C, 52.68; H, 3.45; Br, 25.03; N, 8.76; O, 10.00. $\mathrm{C}_{14} \mathrm{H}_{11} \mathrm{BrN}_{2} \mathrm{O}_{2}$ requires C 52.69; H, 3.47; Br, 25.08; N, 8.78; O, 10.03; IR (KBr) 3400-3200, $3100-2900,2210,1670,1600,1590,1240,1100 \mathrm{~cm}^{-1} ;{ }^{1} \mathrm{H}$ NMR (400 MHz, DMSO-d 6 ) 1.3 (t, 3H, $\left.9.6 \mathrm{~Hz}, \mathrm{CH}_{3}\right), 4.3\left(\mathrm{q}, 2 \mathrm{H}, 9.2 \mathrm{~Hz}, \mathrm{CH}_{2}\right), 7.4(\mathrm{~d}, 1 \mathrm{H}, 11.2 \mathrm{~Hz}), 7.50(\mathrm{~d}, 1 \mathrm{H}, 11.2 \mathrm{~Hz})$, $8.18(\mathrm{~s}, 1 \mathrm{H}), 8.5(\mathrm{~s}, 1 \mathrm{H}, \mathrm{N}-\mathrm{CH}=\mathrm{C}, \mathrm{CH}=\mathrm{C}-\mathrm{CN}), 11.17$ (broad.s); ${ }^{13} \mathrm{C} \mathrm{NMR}(50 \mathrm{MHz})$ : 14.13 $\left(\mathrm{CH}_{3}\right), 61.46\left(\mathrm{OCH}_{2}\right), 93.34,109.49,114.81(\mathrm{CN}), 114.92,117.83,121.33,126.16$, 128.71, 133.35, 134.98, 146.39, 163.01(CO); ES-MS m/z $318\left(\mathrm{M}^{+}, 100 \%\right)$.

\section{5((5-Bromo-3-indolyl)methylene)-2,2-dimethyl-1,3-dioxane-4,6-dione (3h)}

Yellow solid: M.P. 291-292 ${ }^{\circ} \mathrm{C}$; (Found): C, 51.40; H, 3.42; Br, 22.80; N, 4.00; O, 18.28. $\mathrm{C}_{15} \mathrm{H}_{12} \mathrm{BrNO}_{4}$ requires C 51.45; H, 3.45; Br, 22.82; N, 4.00; O, 18.29; IR (KBr) 3400-3200, 31002900, 1700, 1670, 1600, 1590, 1240, $1100 \mathrm{~cm}^{-1} ;{ }^{1} \mathrm{H}$ NMR (400 MHz, DMSO-d $\left.\mathrm{d}_{6}\right), 1.2(\mathrm{~s}, 6 \mathrm{H})$, 7.43(d, 1H, 8.4 Hz), 7.56(d, 1H, 8.4 Hz), 7.96(s, 1H), 8.58(s, 1H, N-CH=C), 9.60(s, 1H, CH=C), 11.3(s, $1 \mathrm{H}, \mathrm{NH}) ;{ }^{13} \mathrm{C}-\mathrm{NMR}(50 \mathrm{MHz}): 14.2\left(2 \mathrm{CH}_{3}\right), 106.42,110.22,115.37,118.45,121.53,125.06$, 127.23, 128.64, 134.22, 136.02, 146.72, 163.64 (2CO); ES-MS m/z 348( $\left.\mathrm{M}^{+}, 100 \%\right)$.

5((5-Bromo-3 indolyl)methylene) pyrimidine-2,4,6 trione (3i)

Yellow solid: M.P. 286-288 ${ }^{0} \mathrm{C}$;(Found): C, 46.71; H, 2.40; Br, 23.89; N, 12.54; O, 14.32. $\mathrm{C}_{13} \mathrm{H}_{8} \mathrm{BrN}_{3} \mathrm{O}_{3}$ requires $\mathrm{C} 46.73 ; \mathrm{H}, 2.41 ; \mathrm{Br}, 23.91 ; \mathrm{N}, 12.58 ; \mathrm{O}, 14.37$; IR (KBr) 3400-3200, 3100-2900, 2210, 1670, 1600, 1590,1240, $1100 \mathrm{~cm}^{-1}$; ${ }^{1} \mathrm{H}$ NMR (400 MHz, DMSO$\left.\mathrm{d}_{6}\right), 7.420(\mathrm{~d}, 1 \mathrm{H}, 8.4 \mathrm{~Hz}), 7.536(\mathrm{~d}, 1 \mathrm{H}, 8.4 \mathrm{~Hz}), 7.99(\mathrm{~s}, 1 \mathrm{H}), 8.58(\mathrm{~s}, 1 \mathrm{H}, \mathrm{N}-\mathrm{CH}=\mathrm{C}), 9.45(\mathrm{~s}, 1 \mathrm{H}$, $\mathrm{CH}=\mathrm{CH}), 11.16$ (b.s, $3 \mathrm{H}, 3 \mathrm{NH}) ;{ }^{13} \mathrm{C}$ NMR $(50 \mathrm{MHz}): 109.27,114.57,118.72,121.33,124.06$, 126.26, 127.02, 134.40, 136.46, 147.92 , 152.40, 164.02 (2CO); ES-MS m/z 332( $\left.\mathrm{M}^{+}, 100 \%\right)$.

\section{2((5-Bromo-3indolyl)methylene)-5,5-dimethylcyclohexane-1,3-dione (3j)}

Yellow solid: M.P. 286-288 ${ }^{0} \mathrm{C}$; (Found): C, 58.95; H, 4.63; Br, 23.06; N, 4.02; O, 9.21. $\mathrm{C}_{17} \mathrm{H}_{16} \mathrm{BrNO}_{2}$ requires $\mathrm{C} 58.97 ; \mathrm{H}, 4.66 ; \mathrm{Br}, 23.08 ; \mathrm{N}, 4.05 ; \mathrm{O}, 9.24$; IR (KBr) 3400$3200,3070,3040,2964,2935,2870,2232,1710,1673,1600,1592,1423,1410,1100 \mathrm{~cm}^{-1}$; ${ }^{1} \mathrm{H}-\mathrm{NMR}(400 \mathrm{MHz}$, DMSO-d 6$), 1.02(\mathrm{~s}, 6 \mathrm{H}), 2.52(\mathrm{~s}, 4 \mathrm{H}), 7.44(\mathrm{~d}, 1 \mathrm{H}, 8.4 \mathrm{~Hz}), 7.52(\mathrm{~d}, 1 \mathrm{H}$, $8.4 \mathrm{~Hz}), 7.95(\mathrm{~s}, 1 \mathrm{H}), 8.57(\mathrm{~s}, 1 \mathrm{H}, \mathrm{N}-\mathrm{CH}=\mathrm{C}), 9.51(\mathrm{~s}, 1 \mathrm{H}, \mathrm{CH}=\mathrm{C}), 11.2(\mathrm{~s}, 1 \mathrm{H}, \mathrm{NH}) ;{ }^{13} \mathrm{C} \mathrm{NMR}$ (50 MHz): 14.62 $\left(2 \mathrm{CH}_{3}\right), 32.53,50.28,110.02,116.27,118.26,122.42,126.42,128.26$, 128.40, 134.65, 135.01, 147.52 ,165.02 (2CO); ES-MS m/z 345( $\left.\mathrm{M}^{+}, 100 \%\right)$.

\section{2((1-Methyl-3indolyl)methylene)malononitrile(3k)}

Yellow solid: M.P. 277-278 ${ }^{0} \mathrm{C}$; (Found): C, 75.31; H, 4.33; N, 20.25. $\mathrm{C}_{13} \mathrm{H}_{9} \mathrm{~N}_{3}$ requires $\mathrm{C}$ 75.35; H, 4.38; N, 20.28; IR (KBr) 3270, 2915, 2843, 2215, 1566, 1495, 1336, 1225, 1139 , $825,789 \mathrm{~cm}^{-1} ;{ }^{1} \mathrm{H}$ NMR(400 MHz, DMSO-d 6 ), 3.98(s, 3H), 7.317-7.375(dt, 2H, J=7.2 Hz ), $7.7(\mathrm{~d}, 1 \mathrm{H}, J=7.6 \mathrm{~Hz}), 7.97(\mathrm{~d}, 1 \mathrm{H}, 7.2 \mathrm{~Hz}) 8.62(\mathrm{~s}, 1 \mathrm{H}), 9.1(\mathrm{~s}, 1 \mathrm{H}) ;{ }^{13} \mathrm{C} \operatorname{NMR}(50$ $\mathrm{MHz},): 33.82\left(\mathrm{~N}_{-} \mathrm{CH}_{3}\right), 73.45,110.24,112.45(2 \mathrm{CN}), 115.24,118.70,122.40,123.45,128.63$, 135.6, 137.01, 150.48; MS-MS $m / z$ 207( $\left.\mathrm{M}^{+}, 100 \%\right)$.

\section{Ethyl-2-cyano-3-(1-methyl-3indolyl)acrylate(3l)}

Yellow crystals: M.P. $264-267{ }^{0} \mathrm{C}$; (Found): C, 70.81; H, 5.54; N, 11.00; O, 12.56. $\mathrm{C}_{15} \mathrm{H}_{14} \mathrm{~N}_{2} \mathrm{O}_{2}$ requires C, 70.85; H, 5.55; N, 11.02; O, 12.58; IR (KBr) 3400-3200, 3100-2900, 2210, 1670, 1600, 1590,1240, $1100 \mathrm{~cm}^{-1} ;{ }^{1} \mathrm{H}$ NMR (400 MHz, DMSO-d 6 ), 1.307(t, 3H, $\left.J=7.2 \mathrm{~Hz}\right), 3.977$ (s, 3H), 4.286(q, 2H, $J=7.2 \mathrm{~Hz}), 7.317-7.375(\mathrm{dt}, 2 \mathrm{H}, J=7.2 \mathrm{~Hz}), 7.637(\mathrm{~d}, 1 \mathrm{H}, J=8 \mathrm{~Hz})$, 7.97(d, $1 \mathrm{H}, 8 \mathrm{~Hz}), 8.522(\mathrm{~s}, 1 \mathrm{H}), 9.01(\mathrm{~s}, 1 \mathrm{H}) ;{ }^{13} \mathrm{C} \mathrm{NMR}(50 \mathrm{MHz}):, 14.15\left(\mathrm{CH}_{3}\right), 33.82\left(\mathrm{~N}_{-} \mathrm{CH}_{3}\right)$, 61.42 $\left(\mathrm{OCH}_{2}\right), 73.04,108.94,111.41,117.80,118.65,122.55,123.75,127.43,135.8,136.96$, 145.82, 163.30(CO); ES-MS m/z 254(M+100\%). 


\section{2,2-Dimethyl-5-((1-methyl-3-indolyl)methylene)-1,3-dioxane-4,6-dione (3m)}

Yellow solid: M.P. $282-283{ }^{0} \mathrm{C}$; (Found): C, 67.35; H, 5.29; N, 4.90; O, 22.40. $\mathrm{C}_{16} \mathrm{H}_{15} \mathrm{NO}_{4}$ requires C, 67.36; H, 5.30; N, 4.91; O, 22.43; IR (KBr) 3400-3200, 3100-2900, 2210, 1670, 1600, 1590, 1240, $1100 \mathrm{~cm}^{-1}$; ${ }^{1} \mathrm{H}$ NMR (400 MHz, DMSO-d $\left.{ }_{6}\right), 1.75(\mathrm{~s}, 6 \mathrm{H}), 3.98(\mathrm{~s}, 3 \mathrm{H}), 7.32(\mathrm{t}$, $1 \mathrm{H}, 7.6 \mathrm{~Hz}), 7.37(1 \mathrm{H}, \mathrm{t}, 7.6 \mathrm{~Hz}) 7.42(\mathrm{~d}, 1 \mathrm{H}, 7.2 \mathrm{~Hz}), 7.97(\mathrm{~d}, 1 \mathrm{H}, 8 \mathrm{~Hz}), 8.61(\mathrm{~s}, 1 \mathrm{H}), 8.7(\mathrm{~s}, 1 \mathrm{H})$; ${ }^{13} \mathrm{C}$ NMR (50 MHz,): 14.20( $\left(\mathrm{CH}_{3}\right), 34.12\left(\mathrm{~N}_{-} \mathrm{CH}_{3}\right), 106.42,110.24,115.24,118.70,122.23$, $123.45,124.02,126.242,135.6,136.02,146.72,163.24(2 \mathrm{CO})$; ES-MS $m / z$ 285(M+1 $100 \%)$.

\section{5-((1-Methyl-3 indolyl)methylene)pyrimidine-2, 4, 6- trione (3n)}

Yellow solid: M.P. $249-251{ }^{0} \mathrm{C}$; (Found): C, 62.41; H, 4.10; N, 15.59; O, 17.80. $\mathrm{C}_{14} \mathrm{H}_{11} \mathrm{~N}_{3} \mathrm{O}_{3}$ requires $\mathrm{C}, 62.45 ; \mathrm{H}, 4.12 ; \mathrm{N}, 15.61$; O, 17.83; IR (KBr) 3400-3200, 3100-2900, 2210, 1670, 1600, 1590,1240, $1100 \mathrm{~cm}^{-1} ;{ }^{1} \mathrm{H}$ NMR (400 MHz, DMSO-d 6 ), 4.01(s, 3H), 7.35(t, 1H, 7.5 $\mathrm{Hz}), 7.39(\mathrm{t}, 1 \mathrm{H}, 7.2 \mathrm{~Hz}), 7.41(\mathrm{~d}, 1 \mathrm{H}, 8 \mathrm{~Hz}), 7.90(\mathrm{~d}, 1 \mathrm{H}, 8 \mathrm{~Hz}), 8.53(\mathrm{~s}, 1 \mathrm{H}), 8.62(\mathrm{~s}, 1 \mathrm{H}), 11.1(\mathrm{~s}$, $2 \mathrm{H}) ;{ }^{13} \mathrm{C}$ NMR $(50 \mathrm{MHz}):, 34.02\left(\mathrm{~N}_{-} \mathrm{CH}_{3}\right), 109.27,112.20,118.62,122.12,123.45,124.24$, 127.02, 136.40, 136.46, 147.02, 152.40(CO),163.24(2CO); ES-MS m/z 269(M+1 $100 \%)$.

\section{5,5-Dimethyl-2-((1-methyl-3indolyl)methylene)cyclohexane-1,3-dione (3o)}

Yellow solid: M.P. 241-243 ${ }^{\circ} \mathrm{C}$; (Found): C, 76.81; H, 6.79; N, 4.97; O, 11.34. $\mathrm{C}_{18} \mathrm{H}_{19} \mathrm{NO}_{2}$ requires 76.84; H, 6.81; N, 4.98; O, 11.37; IR (KBr) 3400-3200, 3070, 3040, 2964, 2935, 2870, 2232, 1710, 1673, 1600, 1592, 1423, 1410, $1100 \mathrm{~cm}^{-1}$; ${ }^{1}$ NMR (400 MHz, DMSO$\left.\mathrm{d}_{6}\right), 1.26(\mathrm{~s}, 6 \mathrm{H}), 2.24(\mathrm{~s}, 4 \mathrm{H}), 3.96(\mathrm{~s}, 3 \mathrm{H}), 7.29(\mathrm{t}, 1 \mathrm{H}, 7.61 \mathrm{~Hz}), 7.35(\mathrm{t}, 1 \mathrm{H}, 7.23 \mathrm{~Hz}), 7.42(\mathrm{~d}$, $1 \mathrm{H}, 8 \mathrm{~Hz}), 7.96(\mathrm{~d}, 1 \mathrm{H}, 8 \mathrm{~Hz}), 8.52(\mathrm{~s}, 1 \mathrm{H}), 8.95(\mathrm{~s}, 1 \mathrm{H}) ;{ }^{13} \mathrm{C} \mathrm{NMR}(50 \mathrm{MHz}):, 14.74\left(2 \mathrm{CH}_{3}\right)$, $32.44\left(2 \mathrm{CH}_{2}\right), 34.22\left(\mathrm{~N}_{-} \mathrm{CH}_{3}\right), 50.28,110.26,112.62,118.62,122.22,123.42,125.01,128.11$, 135.76, 136.66, 148.69,165.40(2CO); ES-MS m/z 281(M+100\%).

\section{2-((1H-indol-3-yl)methylene)malononitrile (3p)}

Yellow solid: M.P.187-189 ${ }^{0} \mathrm{C}$; (Found): C, 74.60; H, 3.65; N, 21.75. $\mathrm{C}_{12} \mathrm{H}_{7} \mathrm{~N}_{3}$ requires C, 74.62; H, 3.67; N, 21.77; IR (KBr) 3270, 2915, 2843, 2215, 1566, 1495, 1336, 1225, 1139, 825, $789 \mathrm{~cm}^{-1} ;{ }^{1} \mathrm{H}$ NMR (400 MHz, DMSO-d $\left.{ }_{6}\right), 7.31(\mathrm{t}, 1 \mathrm{H}, 7.65 \mathrm{~Hz}), 7.36(\mathrm{t}, 1 \mathrm{H}, 7.23)$, 7.54(d, $1 \mathrm{H}, 8 \mathrm{~Hz}), 7.92(\mathrm{~d}, 1 \mathrm{H}, 8 \mathrm{~Hz}), 8.52(\mathrm{~d}, 1 \mathrm{H}), 9.2(\mathrm{~s}, 1 \mathrm{H}), 11.2(\mathrm{~d}, 1 \mathrm{H}) ;{ }^{13} \mathrm{C}$ NMR $(50$ $\mathrm{MHz},): 73.45,110.24,112.45(2 \mathrm{CN}), 115.24,118.70,122.40,123.45,128.63,135.6$, 137.01, 150.48; ES-MS m/z 193( $\left.\mathrm{M}^{+}, 100 \%\right)$.

\section{Ethyl 2-cyano-3-(1H-indol-3-yl)acrylate (3q)}

Yellow Needles: M.P. 160-162 ${ }^{0}$ C; (Found): C, 69.94; H, 5.00; N, 11.64; O, 13.31. $\mathrm{C}_{14} \mathrm{H}_{12} \mathrm{~N}_{2} \mathrm{O}_{2}$ requires C, 69.99; H, 5.03; N, 11.66; O, 13.32; IR (KBr) 3400-3200, 31002900, 2210, 1670, 1600, 1590,1240, $1100 \mathrm{~cm}^{-1} ;{ }^{1} \mathrm{H}$ NMR (400 MHz, DMSO-d 6 ), 1.30(t, 3H, $7.2 \mathrm{~Hz}), 4.27(\mathrm{q}, 2 \mathrm{H}, 7.2 \mathrm{~Hz}), 7.31(\mathrm{t}, 1 \mathrm{H}, 7.6 \mathrm{~Hz}), 7.37(\mathrm{t}, 1 \mathrm{H}, 7.2 \mathrm{~Hz}), 7.63(\mathrm{~d}, 1 \mathrm{H}, 8 \mathrm{~Hz})$, 7.97(d, 1H, $8 \mathrm{~Hz}), 8.52(\mathrm{~s}, 1 \mathrm{H}), 8.91(\mathrm{~s}, 1 \mathrm{H}), 11.1(\mathrm{~s}, 1 \mathrm{H}) ;{ }^{13} \mathrm{C}$ NMR $(50 \mathrm{MHz}):, 14.15\left(\mathrm{CH}_{3}\right)$, 61.42 $\left(\mathrm{OCH}_{2}\right), 73.04,108.94,111.41,117.80,118.65,122.55,123.75,127.43,135.8,136.96$, 145.82, 163.30(CO); ES-MS m/z 240( $\left.\mathrm{M}^{+}, 100 \%\right)$.

\section{3-((2,2-Dimethyl-4,6-dimethylene-1,3-dioxan-5-ylidene)methyl)indole (3r)}

Yellow solid: M.P. $175-176{ }^{\circ} \mathrm{C}$; (Found): C, 66.40; H, 4.80; N, 5.12; O, 23.51. $\mathrm{C}_{15} \mathrm{H}_{13} \mathrm{NO}_{4}$ requires C, 66.41; H, 4.83; N, 5.16; O, 23.59; IR (KBr) 3400-3200, 3100-2910, 2232, 1700, 1670, 1600, 1594, 1240, $1108 \mathrm{~cm}^{-1}$; $^{1} \mathrm{H}$ NMR (400 MHz, DMSO-d 6 ), 1.37(s, 6H), 7.31(t, 1H, 7.6 Hz), 7.37(t, 1H, 7.2 Hz), 7.62(d, 1H, $8 \mathrm{~Hz}), 7.95(\mathrm{~d}, 1 \mathrm{H}, 8 \mathrm{~Hz}), 8.52(\mathrm{~s}, 1 \mathrm{H}), 8.61(\mathrm{~s}$, $1 \mathrm{H}), 11.0(\mathrm{~s}, 1 \mathrm{H}) ;{ }^{13} \mathrm{C}$ NMR (50 MHz,): $14.20\left(\mathrm{CH}_{3}\right), 106.42,110.24,115.24,118.70,122.23$, 123.45,124.02, 126.242, 135.6, 136.02, 146.72,163.24(2CO); ES-MS m/z 271( $\left.\mathrm{M}^{+}, 100 \%\right)$. 
5-((1H-3-indolyl)methylene)pyrimidine-2,4,6trione (3s)

Yellow solid: M.P. $183-187{ }^{\circ} \mathrm{C}$; (Found): C, 61.15; H, 3.52; N, 16.41; O, 18.79. $\mathrm{C}_{13} \mathrm{H}_{9} \mathrm{~N}_{3} \mathrm{O}_{3}$ requires C, 61.18; H, 3.55; N, 16.46; O, 18.81; IR (KBr) 3400-3200, 3100-2900, 2210, 1670, 1600, 1590, 1240, $1100 \mathrm{~cm}^{-1} ;{ }^{1} \mathrm{H}$ NMR (400 MHz, DMSO-d $)$ ) 7.31-7.37(dt, 2H, 7.2 $\mathrm{Hz}, 7.8 \mathrm{~Hz}), 7.62(\mathrm{~d}, 1 \mathrm{H}, 8 \mathrm{~Hz}), 7.93(\mathrm{~d}, 1 \mathrm{H}, 8 \mathrm{~Hz}), 8.52(\mathrm{~s}, 1 \mathrm{H}), 8.62(\mathrm{~s}, 1 \mathrm{H}), 11.0(\mathrm{~s}, 1 \mathrm{H})$, 11.21(s, 2H); ${ }^{13} \mathrm{C}$ NMR $50 \mathrm{MHz}$,): 109.27, 112.20, 118.62, 122.12, 123.45,124.24, 127.02, 136.40, 136.46, 147.02,152.40(CO),163.24(2CO); ES-MS m/z 255(M+, 100\%).

\section{2-((1H-3-indolyl)methylene)-5,5-dimethylcyclohexane-1,3-dione (3t)}

Yellow solid: M.P.162-163 ${ }^{0} \mathrm{C}$; (Found): C, 76.31; H, 6.37; N, 5.21; O, 11.94. $\mathrm{C}_{17} \mathrm{H}_{17} \mathrm{NO}_{2}$ requires C, 76.38; H, 6.41; N, 5.24; O, 11.97; IR (KBr) 3400-3200, 3070, 3040, 2964, 2935, 2870, 2232, 1710, 1673, 1600, 1592, 1423, 1410, $1100 \mathrm{~cm}^{-1} ;{ }^{1} \mathrm{H}$ NMR (400 MHz, DMSO$\left.\mathrm{d}_{6}\right), 1.26(\mathrm{~s}, 6 \mathrm{H}), 2.5(\mathrm{~s}, 4 \mathrm{H}), 7.32-7.37(\mathrm{dt}, 2 \mathrm{H}, 7.2 \mathrm{~Hz}, 7.8 \mathrm{~Hz}), 7.61(\mathrm{~d}, 1 \mathrm{H}, 8.1 \mathrm{~Hz}), 7.92(\mathrm{~d}$, $1 \mathrm{H}, 8.2 \mathrm{~Hz}), 8.52(\mathrm{~s}, 1 \mathrm{H}), 8.62(\mathrm{~s}, 1 \mathrm{H}), 11.1(\mathrm{~s}, 1 \mathrm{H}) ;{ }^{13} \mathrm{C} \mathrm{NMR}(50 \mathrm{MHz}): 14.74\left(2 \mathrm{CH}_{3}\right), 32.44$ $\left(2 \mathrm{CH}_{2}\right), 50.28,110.26,112.62,118.62,122.22,123.42,125.01,128.11,135.76,136.66$, 148.69, 165.40(2CO); ES-MS m/z 267( $\left.\mathrm{M}^{+}, 100 \%\right)$.

\section{Antibacterial activity}

Biological activity of compounds $3 a-3 t$ was studied by disc diffusion technique. Some of the compounds showing the interesting results are tabulated in the Table 2. Most bromo substituted and cyano substituted compounds showed the potent activity against the tested bacteria.

Table 2. Antimicrobial screening results of synthesized indole compounds

\begin{tabular}{ccccccccc}
\hline \multirow{2}{*}{ Compd. } & \multicolumn{3}{c}{ Fungi } & \multicolumn{3}{c}{ Gram(+Ve) bacteria } & \multicolumn{3}{c}{ Gram(-Ve) bacteria } \\
\cline { 2 - 9 } & A.niger & P.notatum & S.aureus & S.faecalis & B.Subtillis & E.coli & K.pneumoniae & S.typhi \\
\hline 3a & +++ & ++ & +++ & - & +++ & + & + & ++ \\
3g & + & - & ++ & - & - & + & + & - \\
3d & - & - & + & + & ++ & - & - & + \\
3i & - & - & +++ & - & +++ & +++ & ++++ & + \\
3l & ++ & - & + & +++ & - & - & + & + \\
\hline
\end{tabular}

Inhibition values beyond control are $+=6-10 \mathrm{~mm},++=11-15 \mathrm{~mm},+++=16-20 \mathrm{~mm}$, $++++=21-25 \mathrm{~mm}$ and $-=$ not active

(The values are including disc diameter); $\mathrm{R}=$ Terbinafin (antifungal agent) and Tetracycline (antibacterial agent). The standards are in the form of sterile Hi-Disc cartridges, each disc containing $10 \mu \mathrm{g}$ of the drug

\section{Acknowledgment}

The authors thank The Head RSIC, CDRI, Lucknow and Fine chemical laboratory, IICT, Hyderabad for NMR and mass spectral data.

\section{References}

1. Mohit L, Deb and Pulak Bhuyan J, Tetrahedron Lett., 2007, 48(12), 2159-2163.

2. Sang-Yun Kim, Pan-Suk Kwon, Tae-Woo Kwon Sung-Kee Chung and Young-Tae Chang, Synthetic Comm., 1997, 27(4), 533-541.

3. VenkatNarsaiah A, Basak A K, Visali B and Nagaiah K, Synthetic Comm., 2004, 34(16), 2893-2901.

4. Hiremath S P, Ashok U, Sekhar K R, and Purohit M G, Indian J Chem., 1988, 27B, 758-762. 
5. Giguere R J, Namen A M, Lopez B O, Arepally A, Ramos D E and Mahetich G, Tetrahedron Lett., 1987, 28(52), 6553.

6. Giguere R.J, Bray T L, Duncan S M and Majetich G, Tetrahedron Lett., 1986, 27(41), 4945-4948.

7. Srikrishna A and Nagaraju S, J Chem Soc Perkin Trans., 1992, 1, 311.

8. Abramovitch R A and Bulman A, Synlett., 1992, 795.

9. (a).Alaharin R, Baquero J J, Garcia Navio J L and Alvarez-Builla J, Synlett,, 1992, 297; (b). Bose A K, Manhas M S, Ghosh M, Raju B S, Tabei K and U - Lipkowska Z, Heterocycles, $1990, \mathbf{3 0}(2), 741$.

10. Ley S V and Mynett D M, Synlett., 1993, 793.

11. Sun W C, Guy P M, Jahngen J H, Rossomando E F and Jahngen E G E, J Org Chem., 1998, 53, 4414-4416.

12. Jahngen E G E, Lentz R R, Pesheck P S and Sackett P H, J Org Chem., 1990, 55, 3406-3409.

13. Bose A K, Banic B K, Barakat K J and Manhas M S, Synlett., 1993, 575.

14. Varma R S, Chatterjee A K and Varma M, Tetrahedron Lett., 1993, 34(29), 4608-4606.

15. Varma R S, Chatterjee A K and Varma M, Tetrahedron Lett., 1993 , 34(20), 3207-3210.

16. Molina A, Vaquero J J, G-Navio J L and A-Builla J A, Tetrahedron Lett., 1993, 34(16), 2673-2676.

17. Qussaid B, Berlan J, Soufiaoui M and Garrigues B, Synthetic Comm., 1995, 25(5), 659-665. 


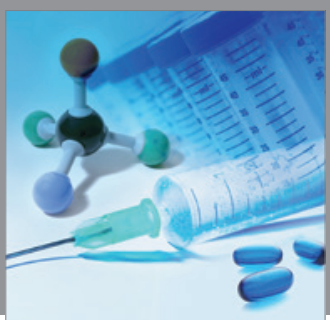

International Journal of

Medicinal Chemistry

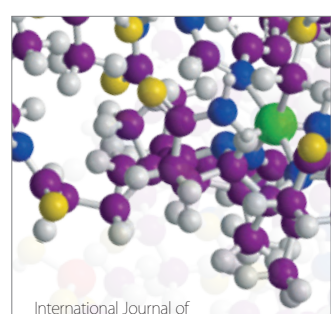

Carbohydrate Chemistry

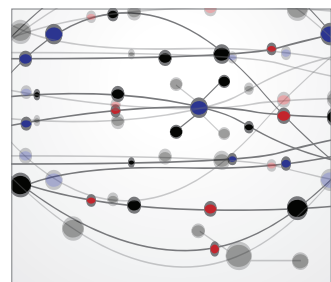

The Scientific World Journal
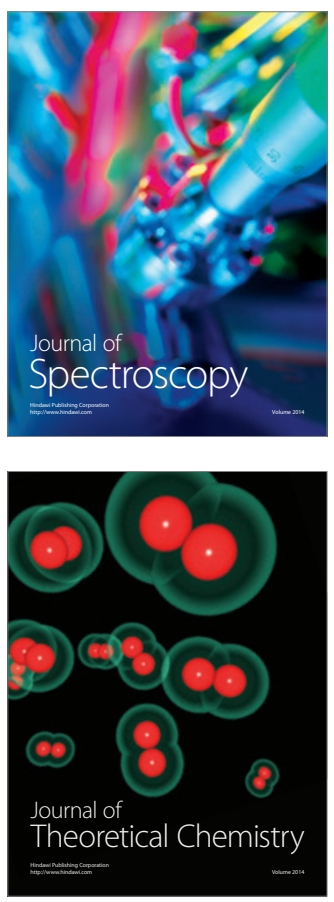
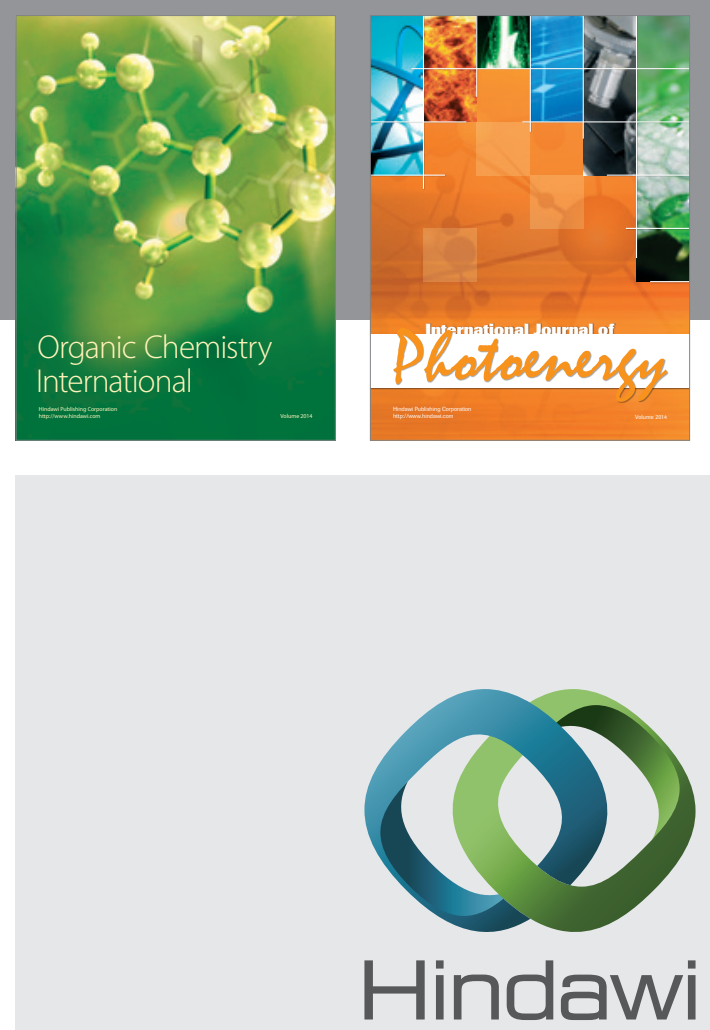

Submit your manuscripts at

http://www.hindawi.com
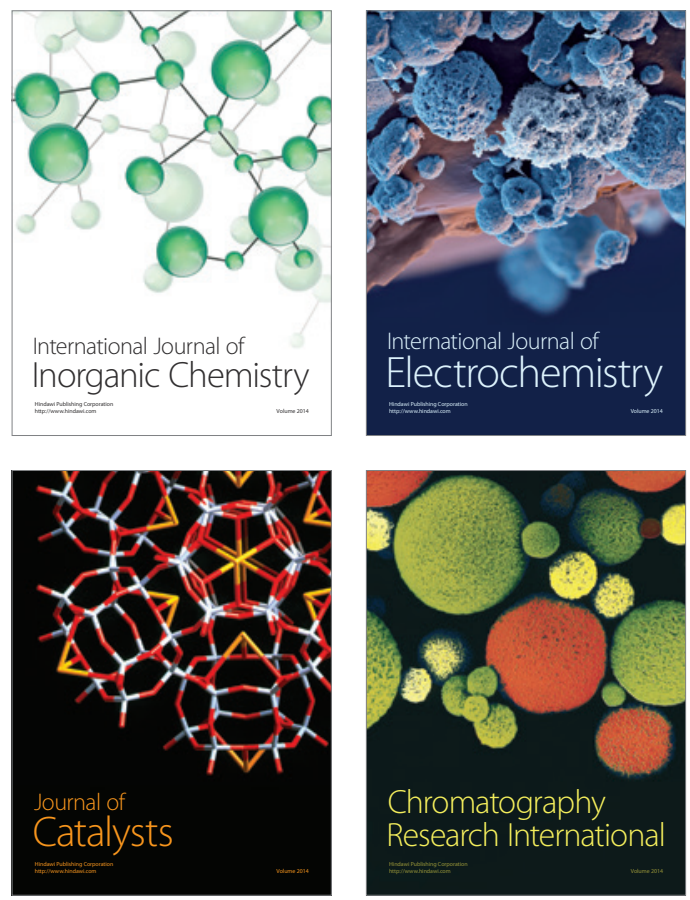
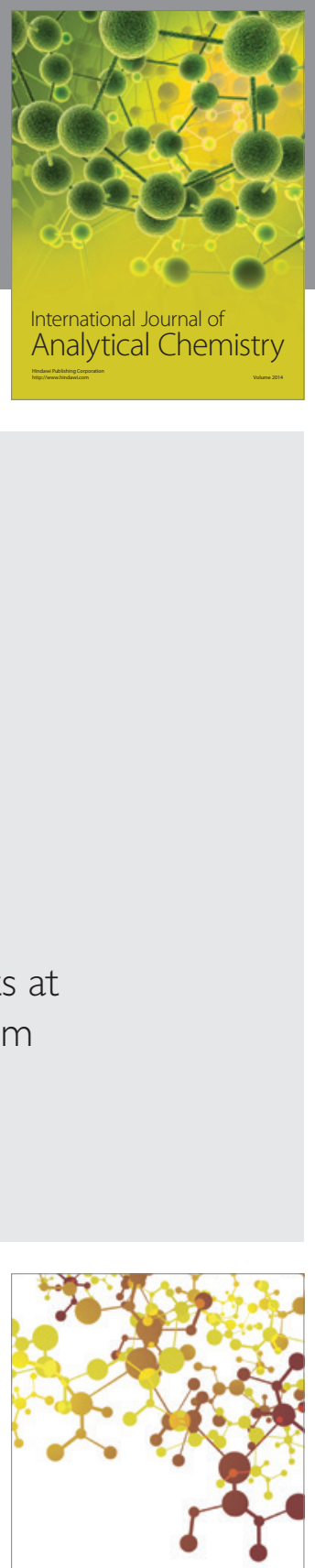

Journal of

Applied Chemistry
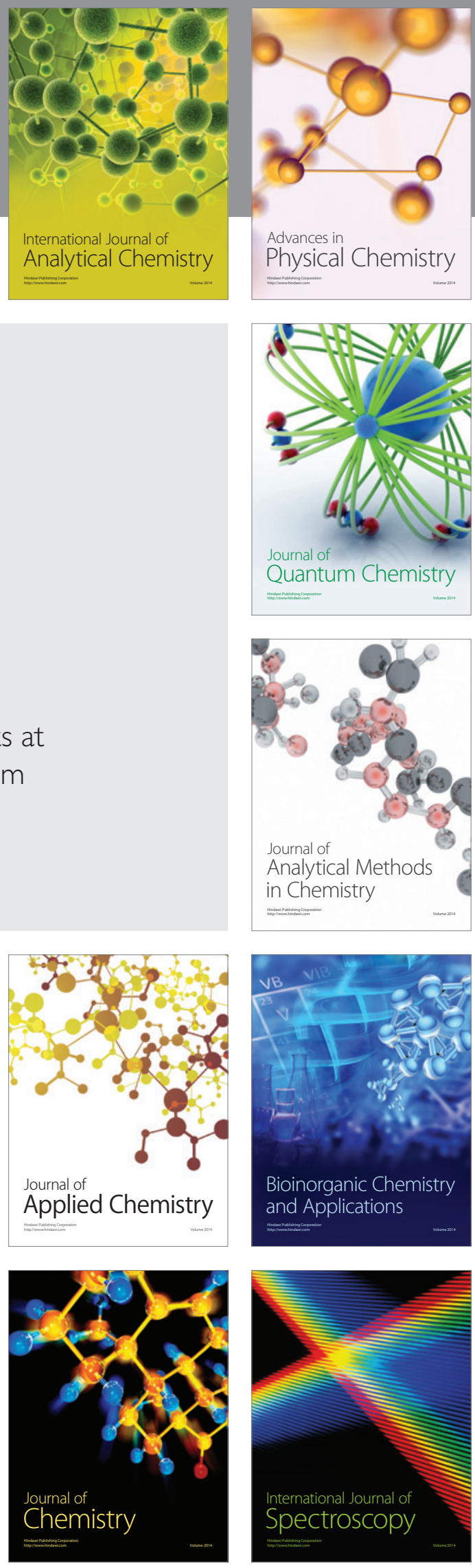